J. H. Bennett, has also incorporated many numerical and typographical corrections as footnotes.

The first volume contains forty-one papers published before 1925. Although during this period statistical theory predominated, Fisher had already manifested a lifelong concern for eugenics and this had stimulated him to make major advances in genetics. The earliest paper, that of 1912 in which he already stated the idea of estimation by maximum likelihood, is an appropriate pointer to a life spent in seeking the optimal use of information. Twenty years later he could assert confidently that "When a biologist believes there is information in an observation, it is up to the statistician to get it out". This volume, of course, includes the great Philosophical Transactions paper and others that so firmly established the theory of statistical inference. Here also the study of exact sampling distributions is taken as far as the various beta and gamma types deriving from the normal distribution. Already Fisher had clarified the analysis of contingency tables through specification of degrees of freedom, and had achieved the simplifications in regression theory basic to so much statistical practice today; the papers are now little known but well deserve attention. Yet again, this period includes the beginnings of the analysis of variance. From the need to interpret the classical Rothamsted experiments, Fisher first devised logical instruments for extracting and organizing the information, then saw how this led to new concepts of experimental design; this series of papers still deserves to be read as a counterbalance to the abstract approach so common today.

Though this giant among statisticians was but one among many great figures in genetics, his contribution to genetics was distinctive, important, and influential. Among these early papers appear some of his first steps to reconcile Darwinism and Mendelism; later he was to do much to show the complementarity of the two. Already he showed his interest both in frequencies at and recombinations between major gene loci and in the biometric genetics of characters dependent upon summed effects of many factors. The mastery with which this young man of 28 , a mathematician untrained in biology, could write "On the Correlation Between Relatives" was a foretaste of the mark he was to make on quantitative genetics; republication of this frequently quoted paper is particularly welcome. The more specifically eugenic papers may now have little scientific value but they throw light on the man; they illustrate the power with which he wrote, his attention both to detail and to grand design, and his care for the shoice of precise word and telling phrase.

This volume is attractive in appearance, pleasant to handle and read, and a credit to both editor and publisher.

D. J. FINNEY

\section{Disks of Drosophila}

The Biology of Imaginal Disks. Edited by $\mathbf{H}$. Ursprung and $\mathbf{R}$. Nothiger. Pp. xvii +172 . (Springer: Berlin and New York, 1972.) 46 DM; $\$ 14.60$.

PROFESSOR HADORN's 70th birthday was preceded by a great deal of surreptitious arrangements with publishers. One of the results is a celebratory volume $T$ he Biology of Imaginal Disks. It is, therefore, a good moment to consider the status of research in this field, and what general problems it can hope to illuminate in the future.

$\mathrm{Up}$ to now, studies on disks have passed through three major periods. In the first era, transplantation, dissociation and reaggregation techniques were used to construct fate maps and to investigate the determined state of different regions of the disks. In the second period the maintenance of the determined state was studied. Here two main discoveries were made, one being the great stability of cell type stemming from even small parts of disks transferred from insect to insect and undergoing hundreds of cell divisions. The other, that at a frequency much higher than that for a typical mutation rate, the cells transformed into cells of another disk (transdetermination). Some transdeterminations turned out to be more probable than others. The third major area, still under active investigation now, is the use of genetic mosaics to analyse those cell interactions which lead to pattern formation in the disks. This method has established that determination and transdetermination are phenomena that occur simultaneously in a group of adjacent cells, which do not themselves constitute a clone.

Currently there are signs of a new developmental genetics analysing visible mutants and lethals by techniques combining clonal analysis with experimental transplantations. The main advantage here is that somatic crossing over can be triggered by $\mathrm{X}$-irradiation at any stage of development chosen by the investigator.

All these matters are well discussed in the book. In addition Fristrom and Oberlander report the development of suitable in vitro methods and isolation procedures to do biochemistry on disks. It may be too much to hope for some elucidation of the biochemical basis of determination, but there is more cause for optimism with this system than with any other I can think of.

The main appeal of the book is that it is very much alive, squarely set in the present time and conveying the excitement in the field. Even if you do not read it until next year it will be more up to date than most books published in 1973. Imaginal disks are a model system for developmental biologists who should find the book fascinating and challenging. Happy birthday, Professor Hadorn.

\section{P. A. LAWRence}

\section{Idiosyncratic Astrophysics}

Dimensional Analysis and Group Theory in Astrophysics. By Rudolf Kurth. Pp. xiv +235. (Pergamon: Oxford and New York, July 1972.) $£ 7.50$.

THIs somewhat idiosyncratic book is interesting but hardly essential reading for workers in the field. In his preface, the author engagingly admits that he had originally envisaged group theory as an underlying theme but that its role in dimensional analysis subsequently appeared less significant. Consequently he has devoted two-thirds of the book to dimensional analysis, with applications to stellar radiation and structure, and the remaining third to reduction of group-invariant systems of differential equations, with application to stellar structure. Examples concerning planets appear in three appendices.

Dimensional analysis is widely used in some branches of physics, but astrophysicists' main use of it seems to be in introductory courses, to give estimates of the order of magnitude of quantities like stellar central temperature. Kurth, however, is trying in this book to make much more serious and extensive use of the technique, and meets with reasonable success. Most of the applications he describes are his own original work. The section on group theory neatly links some well-known methods but contains less that is new.

Kurth's exposition is generally clear and concise, sometimes admirably so, but he occasionally comes irritatingly close to insulting the reader's intelligence. Only once did I find him incomprehensible.

A few specific points should perhaps be recorded. The introductory discussion of the three classical tests of relativity seemed to me top-heavy with assumptions. No reason is given why units of derived quantities are postulated to be monomials of the basic units (which, for example, excludes use of magnitude rather than luminosity). The discussion of the a priori probability distribution function for the constants of proportionality is somewhat unsatisfactory, since the criteria for the choice of such a distribution are not examined. M. A. H. MacCallum 